\title{
Treatment of Lymphoma and Cardiac Monitoring during Pregnancy
}

\author{
Stella Pak, MD \\ Yan Yatsynovich, MD \\ Damian Valencia, MD \\ Department of Medicine \\ Kettering Medical Center \\ Kettering, OH USA
}

\begin{abstract}
Limited data is available regarding fetal-maternal outcomes with chemotherapy during pregnancy, including cardiovascular toxicity and evaluation thereof. Early cardiovascular evaluation and initiation of cardioprotective therapies should be considered. Herein, we report a case of a 33-year-old woman treated with R-CHOP chemotherapy for large Bcell lymphoma found to have some degree of reversible cardiac strain.

\section{Introduction}

There are no guidelines specific for cardiotoxicity monitoring in pregnant patients undergoing chemotherapy. Pregnant patients are more vulnerable to cardiovascular complications, such as congestive heart failure, from chemotherapy as their cardiovascular system is under considerable stress from increasing physiological demands in pregnancy. With elevated cardiac output and circulatory volume from baseline, these patients do not have much cardiopulmonary reserve to compensate for cardiac strains from chemotherapy side effects (1). Therefore, it would be critical for clinicians to be aware of increased risk of cardiovascular adverse effect from chemotherapeutic agents in pregnant patients. Herein, we report a case of a 33-yearold woman treated with R-CHOP chemotherapy for large B-cell lymphoma found to have some degree of reversible cardiac strain.
\end{abstract}

\section{Case Presentation}

An otherwise healthy 33-year-old Caucasian female, G2P1 at 24 weeks gestation presented with a chief complaint of cough, chest pressure, and swelling in the neck and face. Physical exam was notable for a negative Pemberton's sign, two lymph nodes in the right supraclavicular region measuring approximately $2 \mathrm{~cm}$ without axillary or groin lymphadenopathy. Cardiac exam demonstrated distant heart sounds with a faint I-II/VI systolic murmur in the left second intercostal space, without presence of bruits or lower extremity edema. Lung exam was positive for occasional wheezing in the left lower lobe. Breast exam was normal. Initial chest $x$-ray (Figure 1) and computed tomography (CT) scan (Figure 2) of the chest revealed a mediastinal mass $(11 \times 9.2 \times 8.7 \mathrm{~cm})$ and a moderate sized pericardial effusion. 


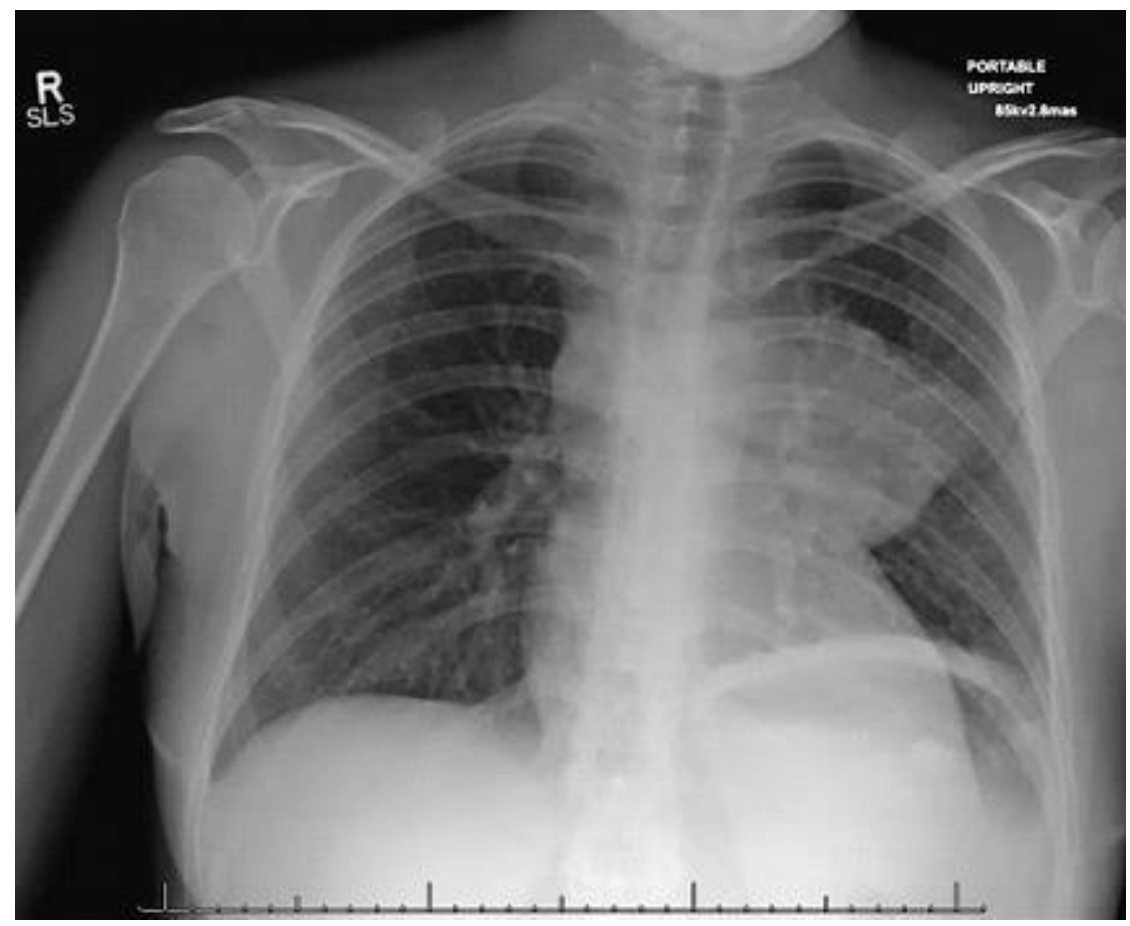

Figure 1. Roentgenogram of chest demonstrating a large mass on left lower lobe and pericardial effusion.

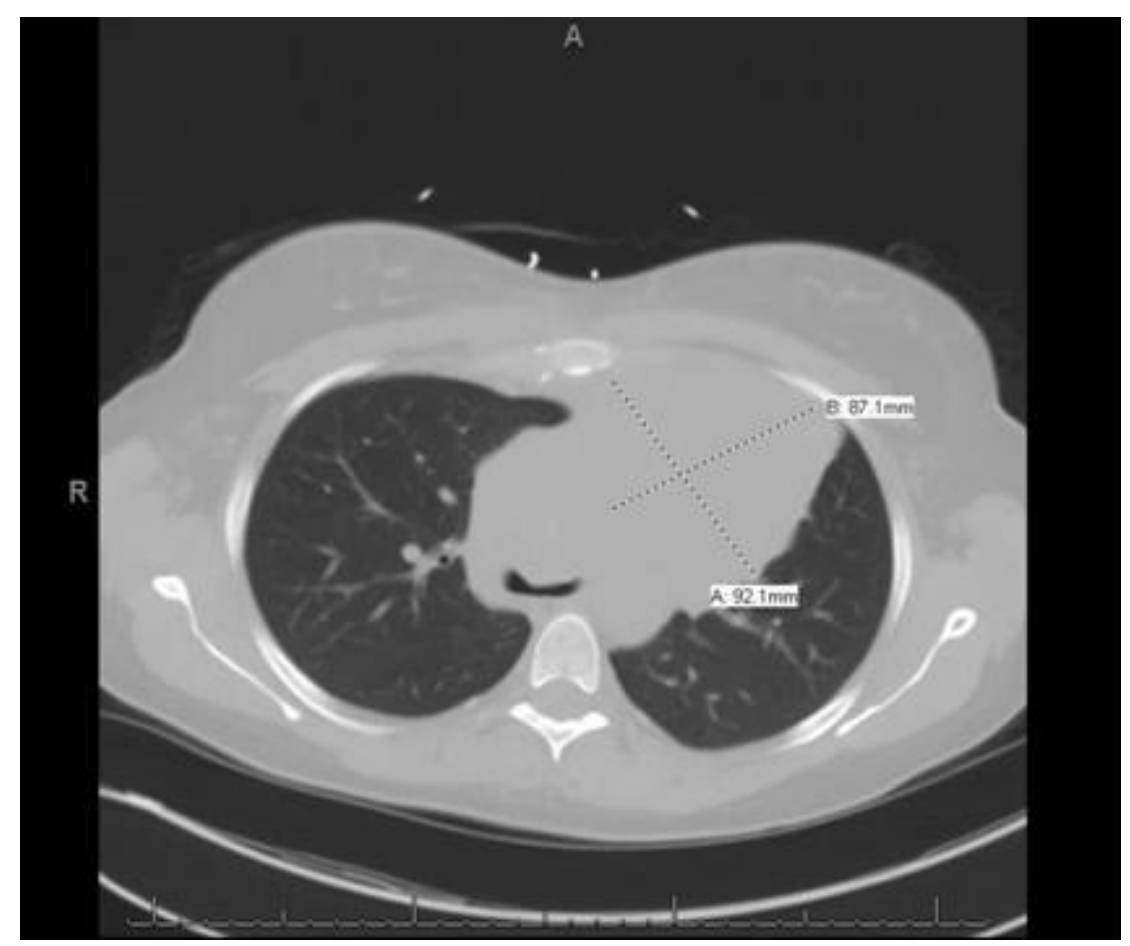

Figure 2. Computerized tomography of chest revealing a large homogeneous left mediastinal mass $(92.1 \mathrm{~mm}$ X $87.1 \mathrm{~mm})$. 
Follow up CT-guided biopsy yielded a diagnosis of large B-cell lymphoma. Bronchoscopy done at that time demonstrated diffuse tracheal and bronchial involvement, likely pointing to primary mediastinal derivation of the tumor. Interestingly, the patient had a history of lymph node biopsy of two areas on her right lateral neck and right medial supraclavicular node; pathology reports were consistent with granulomatous disease at that time. Due to pregnancy, baseline positron emission tomography (PET)/CT was not performed, however, the patient did undergo staging with a CT scan of the chest and abdominal/pelvic and magnetic resonance imaging (MRI), both of which were negative for metastatic disease. Bone marrow biopsy obtained was negative for malignancy as well. Dose-adjusted R-EPOCH (rituximab, etoposide, prednisone, oncovin, cyclophosphamide, hydroxydaunorubicin) was replaced with R-CHOP (rituximab, cyclophosphamide, hydroxydaunomycin, oncovin, prednisolone) due to the teratogenic effects of etoposide. Embryologic toxicity has previously been observed with etoposide including skeletal abnormalities, exencephaly, encephalocele and anophthalmia. Monitoring of cardiac function was performed before, during and after treatment. Initial echocardiogram demonstrated preserved ejection fraction (EF) of $60 \%$ with a large pericardial effusion and early signs of tamponade. No strain studies were done prior to initiation of chemotherapy. The patient had undergone a total of six cycles with a good response. Upon treatment completion fluoro-D-glucose (FDG)-PET/CT did show persistent uptake mostly in the manubrium as well as a persistent mediastinal mass with a low standardized uptake values (SUV).

The patient had initially considered radiotherapy in her post-delivery course, but given her most recent PET scan with a Deauville score of less than 4, the patient decided to avoid radiation and opted for close follow-up with repeat imaging. The Deauville 5-point scoring system is an internationally accepted point based scale used to characterize fluorodeoxyglucose (FDG) avidity of malignant tumor mass as seen on FDG positron emission tomography (PET) scan. Scores between 1 and 2 are considered negative, a score of 3 is typically paired with other studies and clinical signs to determine progression of disease, a score of 4 and 5 are considered positive for malignancy progression. Her pericardial effusion had resolved with chemotherapy.

Echocardiographic cardiac strain evaluation performed during follow-up evidenced a drop in her longitudinal strains from 22.8 to $-15 \%$ just prior to delivery. Ejection fraction remained preserved at $>60 \%$. Low-dose carvedilol was considered during treatment however patient was not agreeable. The patient had an uneventful delivery and strain studies post-delivery showed a stable $-15 \%$ strain. Echocardiogram performed 6 months post-chemotherapy demonstrated an ejection fraction of $72 \%$ and normalization of longitudinal strain. In the light of chemotherapy with known cardiotoxic adverse effects, as well as pregnancy strain on cardiac function, the patient did well and underwent an uneventful course.

\section{Discussion}

The majority of data on maternal and fetal cardiotoxic effects of chemotherapy during pregnancy is based on case reports and retrospective data collection (2). 
Registry data seems to suggest that the incidence of toxic side effects is not significantly increased during pregnancy and in the current literature there is no mention of an increased frequency of heart failure or left ventricular dysfunction during pregnancy (3-5). A study by Van Calsteren et al. (6), suggested that serum levels of chemotherapy, including anthracyclines, measured in pregnant women, were lower compared with those in nonpregnant women although the differences were not statistically significant. Despite the lower serum levels, cardiotoxicity might have a more significant impact on the maternal cardiovascular system in a context of increased hemodynamic loading. The use of cardiotoxic medications during pregnancy requires further attention, however no standard cardiac follow-up protocols are currently in place (7).

There may be a need for clinical cardiac assessments and an echocardiographic functional evaluation, including cardiac strain monitoring, prior to starting chemotherapy and repeat echocardiographic evaluation prior to every dose. If changes in cardiac function are observed, less cardiotoxic treatments might be considered or cardioprotective agents could be used. In this particular patient population, baseline echocardiography with strain study is crucial. Evidence of abnormal strain study during any part of the treatment should prompt initiation of cardioprotective therapy as per standards of the current heart failure guidelines. In addition, we suggest consideration for close cardiac follow-up monitoring, including a repeat echocardiogram study at 12 months post completion of chemotherapy/radiotherapy treatment. It is still unclear whether prophylactic therapy with cardioprotective agents would be safe and beneficial in these patients. Though we may be able to extrapolate data from trials performed on non-pregnant patients undergoing therapy and apply it to this particular niche of patients. The 2013 ACC/AHA heart failure guidelines state that it may be reasonable to evaluate those who are receiving (or who have received) cardiotoxic chemotherapy agents for left ventricular dysfunction as well as use echocardiographic techniques or biomarkers to identify increased heart failure risk in those receiving chemotherapy (8). In addition, the 2012 European Society of Medical Oncology (ESMO) guidelines stress on importance of serials cardiac function monitoring at baseline, 3, 6 and 9 months during treatment and then at 12 and 18 months after initiation of treatment (9).

Today, there is still no clear consensus with regards to cardioprotective therapy in patients exposed to cardiotoxic agents. As of 2016, the ACC/AHA guidelines did not reflect any change in recommendations in this particular field. Risk-stratification and prophylactic cardioprotective therapy remain an ultimate goal in pregnant patients undergoing chemotherapy, but how that should be done is still being studied. Early cardiology involvement and possible early initiation of prophylactic heart failure therapy should be considered.

\section{References}

1. Fadol AP, Lech T, Bickford C, Yusuf SW. Pregnancy in a patient with cancer and heart failure: challenges and complexities. J Adv Pract Oncol. 2012 Mar;3(2):85-93. [PubMed] 
2. Gziri MM, Amant F, Debiève F, Van Calsteren K, De Catte L, Mertens L. Effects of chemotherapy during pregnancy on the maternal and fetal heart. Prenat Diagn. 2012 Jul;32(7):614-9. [CrossRef] [PubMed]

3. Cardonick E, Dougherty R, Grana G, Gilmandyar D, Ghaffar S, Usmani A. Breast cancer during pregnancy: maternal and fetal outcomes. Cancer J. 2010;16(1):76-82. [CrossRef] [PubMed]

4. Van Calsteren K, Heyns L, De Smet F, et al. Cancer during pregnancy: an analysis of 215 patients emphasizing the obstetrical and the neonatal outcomes. J Clin Oncol. 2010 Feb 1;28(4):683-9. [CrossRef] [PubMed]

5. Cardonick $E$, lacobucci $A$. Use of chemotherapy during human pregnancy. Lancet Oncol. 2004 May;5(5):283-91. [CrossRef] [PubMed]

6. Van Calsteren K, Verbesselt R, Ottevanger N, et al. Pharmacokinetics of chemotherapeutic agents in pregnancy: a preclinical and clinical study. Acta Obstet Gynecol Scand. 2010 Oct;89(10):1338-45. [CrossRef] [PubMed]

7. Ewer MS, Ewer SM. Cardiotoxicity of anticancer treatments: what the cardiologist needs to know. Nat Rev Cardiol. 2010 Oct;7(10):564-75. [CrossRef] [PubMed]

8. Yancy CW, Jessup M, Bozkurt B, et al. 20132013 ACCF/AHA guideline for the management of heart failure: executive summary: a report of the American College of Cardiology Foundation/American Heart Association Task Force on practice guidelines. Circulation. 2013 Oct 15;128(16):1810-52. [CrossRef] [PubMed]

9. Curigliano G, Cardinale D, Suter $T$, et al. Cardiovascular toxicity induced by chemotherapy, targeted agents and radiotherapy: ESMO Clinical Practice Guidelines. Ann Oncol. 2012 Oct;23 Suppl 7:vii155-66. [CrossRef] [PubMed] 